\title{
Priority-Aware IP over Hierarchical WDM Ring Networks
}

\author{
Jih-Hsin Ho \\ Department of Leisure \& Information Management, Taiwan Shoufu University, Tainan City, Taiwan
}

\begin{abstract}
WDM (wavelength division multiplexing) appears to be the solution of choice for providing a faster networking infrastructure that can meet the explosive growth of the Internet. It has the scalability problem with increasing the access node numbers in WDM ring architecture, so we further propose the alternatives of hierarchical WDM ring architecture. Since the traditional IP provides the best effort service only, the issue of supporting IP packets with QoS (quality of Service) transfer has become a crucial issue for multimedia transmission. We do so by addressing the priority-aware QoS model. Finally present performance analysis multiple classes of hierarchical WDM ring networks.
\end{abstract}

Key words: Hierarchical WDM ring, priority-aware, performance analysis.

\section{Introduction}

With the explosion of information traffic due to the Internet, electronic commerce, computer networks, voice, data, and video, the need for a transmission medium with the bandwidth capabilities for handling such a vast amount of information is paramount. Recently, the channel bandwidth of commercial WDM (Wavelength Division Multiplexing) communication system has reached to OC-192 (10 Gbps) [1], and the total bandwidth of an optical fiber exceeds 1 Tbps. This indicates that WDM is the solution for bandwidth insatiability.

To increase network bandwidth and decrease packet transmission delay are current important studies due to the internet applications are popular and the bandwidth demands are raised, which can improve effectively by adding fiber channels and switch speed techniques. Moreover using the hierarchical networks decrease the diameters and average distances are worth to study topics, especially the networks exceed the thousand nodes. The hierarchical ring network architectures can decrease the network diameters [2-4].

HWDM-Ring (hierarchical wavelength division

Corresponding author: Jih-Hsin Ho, Ph.D., assistant professor, research fields: performance evaluation, WDM networks, Internet, QoS. E-mail: hjsin@tsu.edu.tw. multiplexing ring) networks are ring network architectures connected by fibers. Fig. 1 shows a diagram of three-level HWDM-Ring where all nodes connected two different rings which are connected rings and local rings. Processing nodes (Access nodes) connected by local rings which are connected by connected rings, and multiple connected rings are connected by higher level connected rings that achieve the hierarchical architectures. Routing nodes (Core nodes) are communicational interface connected between different level rings. Local rings are composed some processing node and one routing node and connected rings are composed all routing nodes. For example HWDM-Ring shown in Fig. 1 is formed by two level connected rings and one level local ring and one ring has 13 processing nodes and whole three-level HWDM-ring has 234 processing nodes. The routing nodes or processing nodes in the same connected rings or local rings own different wavelength using the WDM access technology.

Since the traditional IP provides the best effort service only, the issue of supporting IP packets with QoS (quality of Service) transfer has become a crucial issue for multimedia transmission. Today, while the network bandwidth has grown dramatically, the kind of applications transferred are mostly high-bandwidth demanding multimedia transmissions. It is predictable 


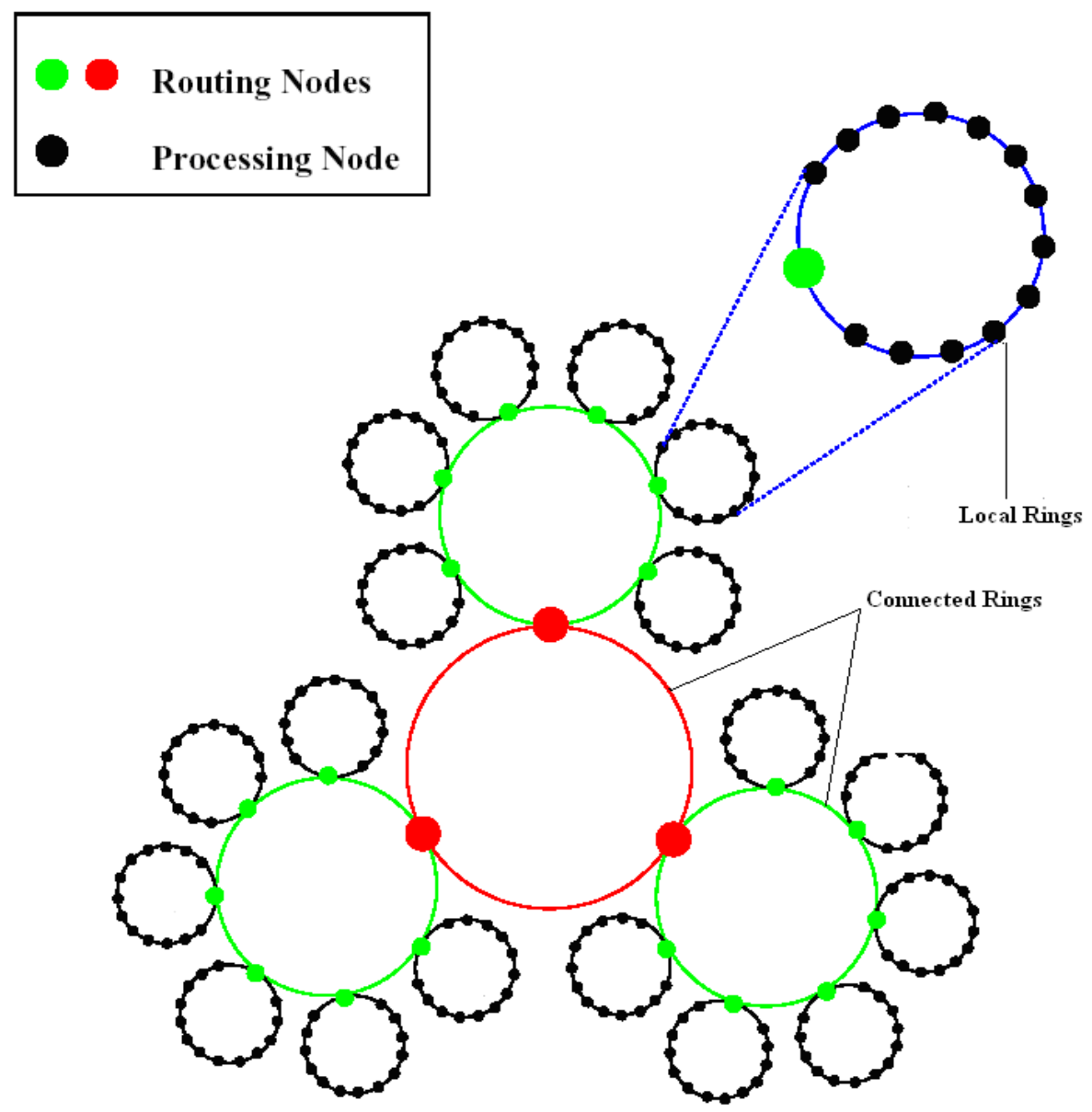

Fig. 1 The example of hierarchical WDM ring networks.

that the end-to-end QoS will be an important area of study in the next generation Internet.

The rest of this paper is organized as follows. The hierarchical WDM ring and node architectures are presented in Section 2. Section 3 introduces the priority-aware mechanism. In Section 4, an approximate queue model for this priority-aware protocol is presented to evaluate the performance. In Section 5, the numerical results are obtained from our analysis. Concluding remarks are made in Section 6.

\section{Hierarchical WDM Ring and Node Architectures}

Hierarchical WDM ring networks are comprised a number of levels which included many ring networks. Higher level rings are connected by routing node in the lower level rings. The processing nodes are located on lowest level and every ring has a routing node exactly connected by upper level ring besides the highest ring.

Using HWDM-Ring $\left(\mathrm{B}_{1}, \mathrm{~B}_{2}, \ldots \mathrm{B}_{\mathrm{i},}, \ldots, \mathrm{B}_{\mathrm{m}}\right)$ to present a m-level hierarchical ring included $\prod_{i-1}^{m} B i$ processing nodes and $\sum_{i=1}^{m-1} \coprod_{j=1}^{i} B_{j}$ routing nodes. Take Fig. 1 for example, this diagram presents HWDM-Ring $(3,6,13)$ which is 3-level hierarchical ring and included 234 processing nodes and 21 routing nodes.

Network diameter is defined as the maximum distance between the nodes arbitrarily. In the WDM single-ring network which has $\mathrm{n}$ nodes and the diameter is $\mathrm{n}-1$. In the HWDM-Ring $\left(\mathrm{B}_{1}, \mathrm{~B}_{2}, \ldots \mathrm{B}_{\mathrm{i}}, \ldots, \mathrm{B}_{\mathrm{m}}\right)$ network, the diameter is $\left(B_{1}-1\right)+2 \cdot \sum_{i=2}^{m} B_{i}$. 
Giving the HWDM-Ring $\left(\mathrm{B}_{1}, \mathrm{~B}_{2}, \ldots \mathrm{B}_{\mathrm{i}}, \ldots, \mathrm{B}_{\mathrm{m}}\right)$ network which every node has a m-tuple address $\mathrm{A}\left(\mathrm{a}_{1}, \mathrm{a}_{2}, \ldots, \mathrm{a}_{\mathrm{k}}, \ldots \mathrm{a}_{\mathrm{m}}\right)$ means this node address located on this ring. The routing node address $\left(\mathrm{a}_{1}, \mathrm{a}_{2}, \ldots, \mathrm{a}_{\mathrm{k}}, 0,0, \ldots 0\right)$ stands for the composed the source node address $\left(\mathrm{a}_{1}, \mathrm{a}_{2}, \ldots, \mathrm{a}_{\mathrm{k}}, 1,0, \ldots 0\right)$, $\left(a_{1}, a_{2}, \ldots, a_{k}, 2,0, \ldots 0\right), \quad \ldots \quad\left(a_{1}, a_{2}, \quad \ldots, a_{k}, B_{k+1}, 0, \ldots 0\right)$. Giving the source node address $S\left(s_{1}, s_{2}, \ldots, s_{m}\right)$ and the destination node address $\mathrm{T}\left(\mathrm{t}_{1}, \mathrm{t}_{2}, \ldots, \mathrm{t}_{\mathrm{m}}\right)$. The routing rule finds the path to transmit data packet from source node to the destination node. In this routing rule finds the routing node from the source node, to find the lower level routing node once more and search the highest level routing node. Inversely from the highest level routing node to find the destination node located on lowest level. For example, the 3-level hierarchical ring has source node address $(0,2,6)$ and destination node address $(2,4,9)$, we find the routing path using the routing rule is followed by $(0,2,6),(0,2,7),(0,2,8)$, $(0,2,9),(0,2,10),(0,2,11),(0,2,12),(0,2,13),(0,2,0)$, $(0,3,0),(0,4,0),(0,5,0),(0,6,0),(0,0,0),(1,0,0),(2,0,0)$, $(2,1,0),(2,2,0),(2,3,0),(2,4,0),(2,4,1),(2,4,2),(2,4,3)$, $(2,4,4),(2,4,5),(2,4,6),(2,4,7),(2,4,8)$ and $(2,4,9)$.

The node included processing and routing nodes architecture of the network is shown in Fig. 2. Each node has one tunable transmitter and $\mathrm{W}$ fixed receivers with one for each data channel. For the optical signal sent from upstream nodes, a splitter is used to tap off a small portion of the optical power from the ring to the receivers. Every receiver detects the optical signal carried in its corresponding wavelength within the output branch from the splitter for node address identification. If the destination address in the incoming packet header matches the node address, the packet data are sent to the host. Meanwhile, the MAC control scheme is signaled to activate the opening of the on-off switch for the corresponding data channel in order to remove the received packet carried in the major portion of the optical signal through the delay line. If the packet is not destined to this node, the detected packet is ignored and the process of scanning next the new packet is started.

\section{Priority-Aware Mechanism}

In the current Internet, the same-service-fit-all paradigm, which may cause serious network congestion and packet loss, does not suit real-time multimedia

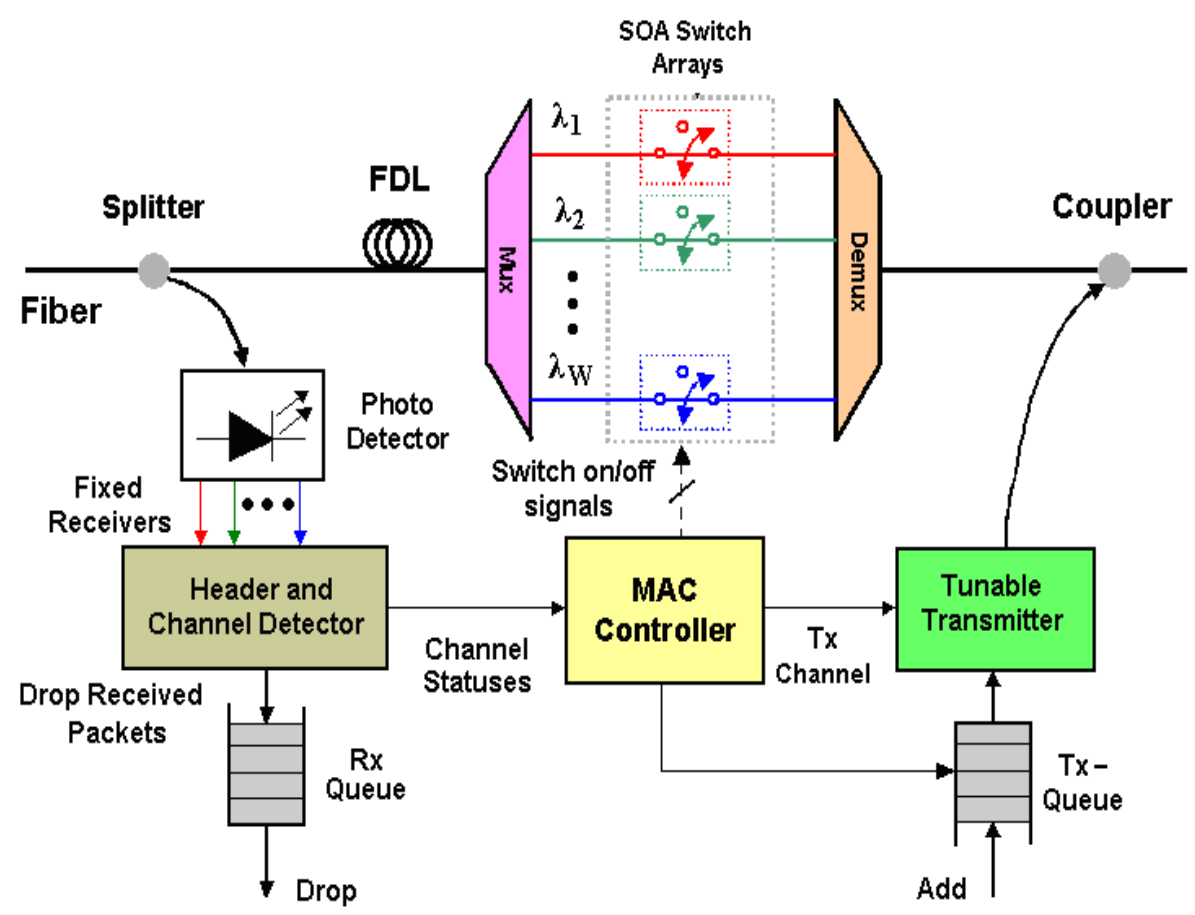

Fig. 2 Node architecture. 
applications, such as video conferencing, Internet phone and video on demand. In order to support these multimedia applications to operate properly and provide them with the adaptive service-level conditions, the issue of QoS has received increasing attention. The priority-aware mechanism adds a QoS function to the transmission queue (TX Queue), is based on the hierarchical CSMA/ID MAC protocol [5] which has been discussed previously. By marking the ToS (type of service) field within the IP packet header, the access node could identify the types of prioritized packet forwarding for each class of service.

\section{Network Performance}

From the behavior of a priority-aware queue model, the model can be categorized as a non-preemptive priority M/G/1 queue model [6]. Note that the $\rho_{i, k}$ is the product of $\lambda_{i, k}$ ( individual class $k$ arrival rate in node $i$ and $\sum \lambda_{i, k}=\lambda_{i}$ ) by $\mathrm{E}\left[X_{i, k}\right]$ (the mean service time of class $k$ in node $i$ ). The notation $\gamma$ is the product of $\mathrm{E}\left[n_{G}\right]$ by $\mathrm{E}[d]$.

Fig. 3 shows model of corresponding priority $k$ in access node $i$. Considering the mean waiting time $W_{i, 1}$ of the highest priority queue, we obtain:

$$
\begin{aligned}
W_{i, 1} & =\alpha+\lambda_{i, 1} \cdot W_{i, 1} \cdot E\left[X_{i, 1}\right]+\lambda_{i, 1} \cdot W_{i, 1} \cdot E\left[n_{G}\right] \cdot E[d] \\
& =\frac{\alpha}{1-\lambda_{i, 1} \cdot E\left[X_{i, 1}\right]-\lambda_{i, 1} \cdot E\left[n_{G}\right] \cdot E[d]} \\
& =\frac{\alpha}{1-\rho_{i, 1}-\lambda_{i, 1} \cdot \gamma}
\end{aligned}
$$

For the second priority queue, we have a similar expression for the mean waiting time $W_{i, 2}$, except that we have to count the additional waiting time due to packets of higher priority that arrive while a packet is

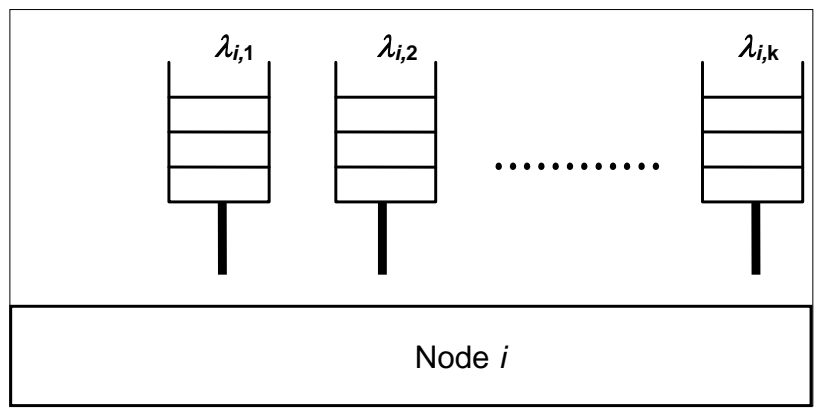

Fig. 3 Queuing model of corresponding priority $k$ in access node $i$. waiting in queue. Using the Eq. (1) obtained earlier, we finally have:

$$
\begin{aligned}
W_{i, 2}= & \alpha+\lambda_{i, 1} \cdot W_{i, 1} \cdot E\left[X_{i, 1}\right]+\lambda_{i, 1} \cdot W_{i, 1} \cdot E\left[n_{G}\right] \cdot E[d] \\
& +\lambda_{i, 2} \cdot W_{i, 2} \cdot E\left[X_{i, 2}\right]+\lambda_{i, 2} \cdot W_{i, 2} \cdot E\left[n_{G}\right] \cdot E[d] \\
& +\lambda_{i, 1} \cdot W_{i, 2} \cdot E\left[X_{i, 1}\right]+\lambda_{i, 1} \cdot W_{i, 2} \cdot E\left[n_{G}\right] \cdot E[d] \\
& =\frac{\alpha+\lambda_{i, 1} \cdot W_{i, 1} \cdot E\left[X_{i, 1}\right]+\lambda_{i, 1} \cdot W_{i, 1} \cdot E\left[n_{G}\right] \cdot E[d]}{1-\lambda_{i, 2} \cdot E\left[X_{i, 2}\right]-\lambda_{i, 2} \cdot E\left[n_{G}\right] \cdot E[d]-\lambda_{i, 1} \cdot E\left[X_{i, 1}\right]-\lambda_{i, 1} \cdot E\left[n_{G}\right] \cdot E[d]}(2) \\
& =\frac{\alpha+\rho_{i, 1} \cdot W_{i, 1}+\lambda_{i, 1} \cdot W_{i, 1} \cdot \gamma}{1-\left(\rho_{i, 1}+\rho_{i, 2}\right)-\left(\lambda_{i, 1}+\lambda_{i, 2}\right) \cdot \gamma} \\
& =\frac{\alpha}{\left(1-\rho_{i, 1}-\lambda_{i, 1} \cdot \gamma\right)\left(1-\left(\rho_{i, 1}+\rho_{i, 2}\right)-\left(\lambda_{i, 1}+\lambda_{i, 2}\right) \cdot \gamma\right)}
\end{aligned}
$$

The derivation is similar for the general priority queue (class $k>1$ ). The formula for the mean waiting time in queue is:

$$
W_{i, k}=\frac{\alpha}{\left(1-\sum_{l=1}^{k-1} \rho_{i, l}-\left[\sum_{l=1}^{k-1} \lambda_{i, l}\right] \cdot \gamma\right) \cdot\left(1-\sum_{l=1}^{k} \rho_{i, l}-\left[\sum_{l=1}^{k} \lambda_{i, l}\right] \cdot \gamma\right)}
$$

\section{Results and Discussions}

In this section, we will present the analytical and simulation results of packet transfer delay of the network. To evaluate and compare the performance of the hierarchical WDM ring network and WDM single-ring network, the following parameters have been listed below.

The simulation experiments are based on the codes by SIMSCRIPT II and are replicated corresponding to variance reduction technique with different sequences for pseudo random numbers. The results are obtained with $95 \%$ confidence level.

The WDM single-ring network:

- Number of processing nodes 20;

- Number of channels 4;

- Propagation delay between adjacent nodes $14 \mu \mathrm{sec}$;

- Channel speed 10 Gbps';

- Size of the delay line 800 bits;

- Average IP packet size 512 bytes.

The hierarchical WDM ring network:

- 3-level HDWM-Ring $(2,2,5)$ with the number of processing nodes 20 and routing nodes 6 ;

- Number of channels 4; 


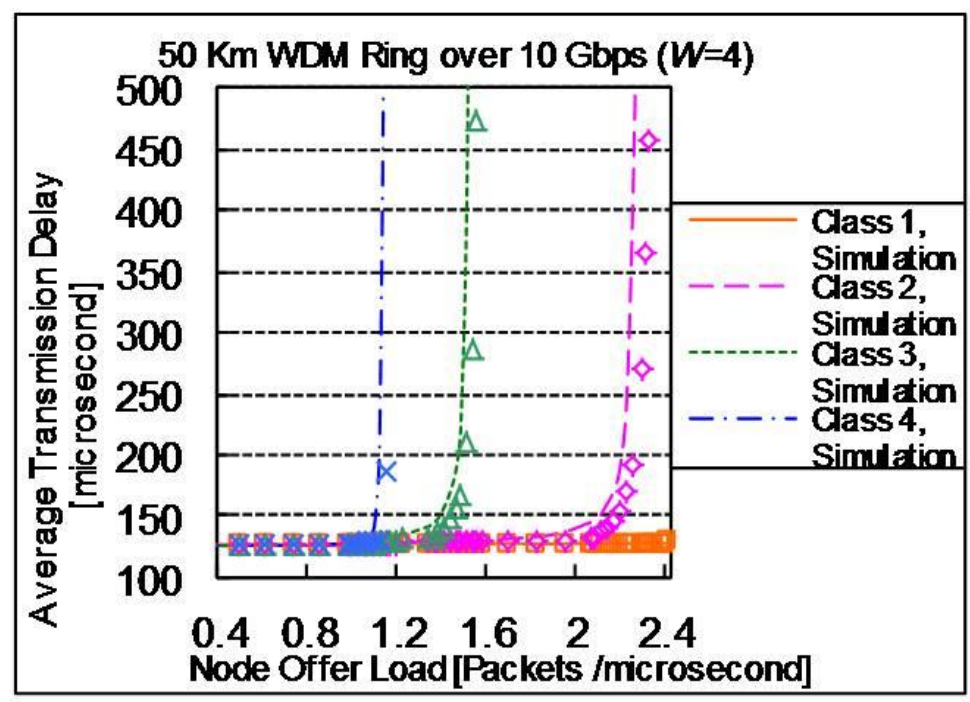

Fig. 4 Average transmission delay under different class $(k=1,2,3$ and 4) using priority-aware model.

- Propagation delay between adjacent nodes 14 usec;

- Channel speed 10 Gbps;

- Size of the delay line 800 bits;

- Average IP packet size 512 bytes.

In the assumption, there are 20 processing nodes with attached to four wavelengths $(W=4)$, and IP packets are divided into four classes. The total traffic is divided equally to each class, i.e., a quarter of the total packet amount. Fig. 4 presents the different performance for each class under the priority-aware model, where Class 4 packets get the worst quality of service and Class 1 packets get the best quality of service. The performance between each service class has a notable diversity. Moreover the agreement between the simulation results and the analytical results is excellent.

\section{Conclusions}

In summary, in this paper we have investigated a hierarchical WDM ring network. The proposed priority-aware mechanism adds the class-based priority scheme into hierarchical WDM ring networks. This novel protocol may differentiate traffic, so when used to implement IP links it is able to help the access nodes implement the QoS-aware (quality-of-service-aware) communication needed in a network that carries multimedia traffic. It also derives the approximate equations for the average packet transfer delay for priority-aware mechanism. For verification, a simulation program obtains simulated results for the network, and the results closely resemble the analytical values, and this demonstrates the good performance of the network. It is also observed that the throughput characteristic of the network is almost proportional to the number of channels in the network. Transfer delay improves with the number of wavelengths and QoS improves with the class-based priority scheme used in the ring, consistent with current WDM technology trends.

\section{References}

[1] Veitch, P. 2002. "Resilience for IP over DWDM Backbone Networks." Electronics \& Communication Engineering Journal 14: 39-48.

[2] Dandamudi, and Eager, D. 1990. "Hierarchical Interconnection Networks for Multicomputer Systems." IEEE Transactions on Computers 39: 786-97.

[3] Dowd, P., Bogineni, K., Aly, K. A., and Perreult, J. A. 1993. "Hierarchical Scalable Photonic Architectures for High Performance Processor Interconnection." IEEE Transactions on Computers 42: 1105-20.

[4] Louri, A., and Gupta, R. 1997. "Hierarchical Optical Interconnection Network (HORN): Scalable 
Interconnection Network for Multiprocessors and Multicomputers." Applied Optics 36: 430-42.

[5] Ho, J. H. 2013. "Performance Evaluation of IP over
Hierarchical CSMA/ID WDM Ring Networks." Journal of Communication and Computer 10: 325-31.

[6] Kleinrock, L. Priority Queueing, Queueing System II. 